\title{
Role of the CS1 adhesion motif of fibronectin in T cell adhesion to synovial membrane and peripheral lymph node endothelium
}

\author{
A C H M van Dinther-Janssen, S T Pals, R J Scheper, C J L M Meijer
}

\begin{abstract}
Objectives-It has previously been shown that the very late antigen-4/vascular cell adhesion molecule-1 (VLA-4/VCAM-1) pathway functions as a receptor/ligand interaction system mediating the recruitment of activated lymphocytes to inflamed synovium of patients with rheumatoid arthritis. This study was performed to determine whether VLA-4 also affects lymphocyte adhesion to inflamed synovium through interaction with the alternatively spliced CS1 domain of fibronectin.
\end{abstract}

Methods-The effect of the synthetic peptide CS1 on lymphocyte binding to human synovial and peripheral lymph node high endothelial venules (HEVs) was measured in an in vitro frozen section assay.

Results-In the presence of the CS1 peptide or antibody to fibronectin, significant inhibition of binding was observed (54 and 51\% respectively). Blocking with antibody to VCAM-1 yielded inhibition of binding to $46 \%$ of the control value. Maximum inhibition of binding was obtained with a combination of antibody to VCAM-1 and CS1 $(65 \%)$ and with antibody to VLA-4 $\alpha(68 \%)$. Blocking the classical fibronectin receptor with antibody to VLA-5 $\alpha$ gave a slightly lower inhibition at $42 \%$. In normal peripheral lymph nodes, the synthetic peptide CS1 and antibodies to fibronectin and VLA-5 also partially inhibited $T$ cell binding to HEVs $(45,47$, and $52 \%$ respectively).

Conclusion-These results show that fibronectin mediates lymphocyte-HEV interactions not only through its classical VLA-5 receptor, but also through its CS1 adhesion motif in inflamed synovium and peripheral lymph nodes.

(Ann Rheum Dis 1993; 52: 672-676)

Lymphocyte recirculation between the blood and the secondary lymphoid organs is mediated by leucocyte-endothelial interactions in which adhesion molecules called 'homing receptors' play an important part. ${ }^{1-5}$ Although the molecular mechanisms underlying lymphocyte high endothelial venule (HEV) recognition are not fully unravelled, two structurally and functionally distinct pathways have been described-that is, the lymphocyte function associated antigen-1 (LFA-1)/intercellular adhesion molecule-1 (ICAM-1) and the very late antigen-4 (VLA-4)/vascular cell adhesion molecule-1 (VCAM-1) pathways. $^{6-9}$ The two pathways are mediated through the interactions of members of the integrin family-that is, LFA-1 and VLA-4-with members of the immunoglobulin superfamily - that is ICAM-1 and VCAM-1. ${ }^{10} 11$ The LFA-1/ICAM-1 pathway has been shown to affect lymphocyte recirculation and homing in a non-organ specific way, ${ }^{12}{ }^{13}$ whereas the VLA-4/VCAM-1 pathway plays an important part in lymphocyte migration into inflamed tissues. ${ }^{14}$ It has been recognised that VLA-4 not only functions as a cell receptor for VCAM-1 ${ }^{9}{ }^{15}$ but also as an extracellular matrix receptor for an alternatively spliced domain of fibronectin (CS1). ${ }^{16-18}$ As in rats CS1 has been found to support the adhesion of lymphocytes to cultured peripheral lymph node (PLN)-high endothelial cells, ${ }^{19}$ we wanted to establish whether CS1 also mediates lymphocyte-HEV interactions in humans. Expression of functional HEV binding ability was tested in a modified in vitro frozen section assay in which lymphocytes bind to HEVs of PLNs or inflamed synovial membranes. The results show that the CS1 adhesive motif of fibronectin affects physiological lymphocyte recirculation via PLN as well as lymphocyte migration to inflamed synovial tissue.

\section{Materials and methods}

PEPTIDES

The CS1 peptide was used to investigate involvement of the type III connecting segment (IIICS) cell binding domain of fibronectin in lymphocyte adhesion. The adjacent peptide sequence (CS2) was used as a control. Peptides were synthesised and purified as described by Humphries et al. ${ }^{18}$ As the maximum inhibition of $\mathrm{T}$ lymphocyte-HEV binding was obtained using concentrations exceeding $0.10 \mathrm{mg}$ peptide/ $/ \mathrm{ml}$, a concentration of $0.15 \mathrm{mg} / \mathrm{ml}$ was used throughout the study.

MONOCLONAL ANTIBODIES

The monoclonal antibodies used were CLB-45 (IgG1) specific for $\mathrm{CD}_{4} 5^{20}$; $\mathrm{HP} 2 / 1$ (IgG1) and SAN1 (IgG1) specific for the $\alpha$ chain of VLA4 (CD49d) and VLA-5 (CD49e) respectively $^{21}{ }^{22}$; 4B9 (IgG1) specific for VCAM-1 ${ }^{23}$; and a rabbit polyclonal antibody against fibronectin (Dakopatts, Glostrup, Denmark). 
IN VITRO HEV BINDING ASSAY

The in vitro binding of human lymphocytes to HEVs was measured by the modified frozen section assay as described previously. ${ }^{14}$ Briefly $1 \times 10^{6}$ lymphocytes were incubated with mild rotation for 30 minutes at $4^{\circ} \mathrm{C}$ on freshly cut frozen sections of PLNs or synovial membranes of patients with rheumatoid arthritis (RA). After incubation the sections were fixed in cold phosphate buffered saline containing $2 \%$ glutaraldehyde and briefly stained with $1 \%$ toluidine blue in aqua dest to distinguish lymphocytes from monocytes. The HEV adherent cells were measured for each standard unit of HEV length by a graphic tablet fitted to a microcomputer (MOB-videoplan, Kontron, Eching, Germany) as described previously. ${ }^{14}$ In inhibition studies lymphocytes and tissue sections were preincubated for 30 minutes at $4^{\circ} \mathrm{C}$ with peptides or monoclonal antibodies which were then stored in the medium during the adhesion assay. Concentrations of the blocking antibodies used were based on titration studies to obtain maximum inhibitory effects. The tissues used were synovial membranes from joints of patients with classic or definite RA according to the criteria of the American Rheumatism Association and were obtained during reconstructive joint operations. Non-rheumatoid synovial membranes were obtained from amputated limbs of patients with vascular insufficiency. In addition, normal or reactive PLNs were used.

\section{ISOLATION OF LYMPHOCYTES}

Peripheral blood T lymphocytes (ten samples) were isolated by Ficoll Hypaque (Pharmacia Uppsala, Sweden) density gradient centrifugation. Monocytes were removed by adherence to plastic Petri dishes (Falcon Plastics, Oxnard, CA, USA) for one hour at $37^{\circ} \mathrm{C}$ and the nonadherent cells were depleted of $\mathbf{B}$ cells by a panning technique. ${ }^{13} \mathrm{~T}$ cell enriched populations contained $>95 \% \mathrm{CD} 2+$ cells and $<5 \%$ surface $\mathrm{Ig}+$ cells as determined by immunofluorescence.

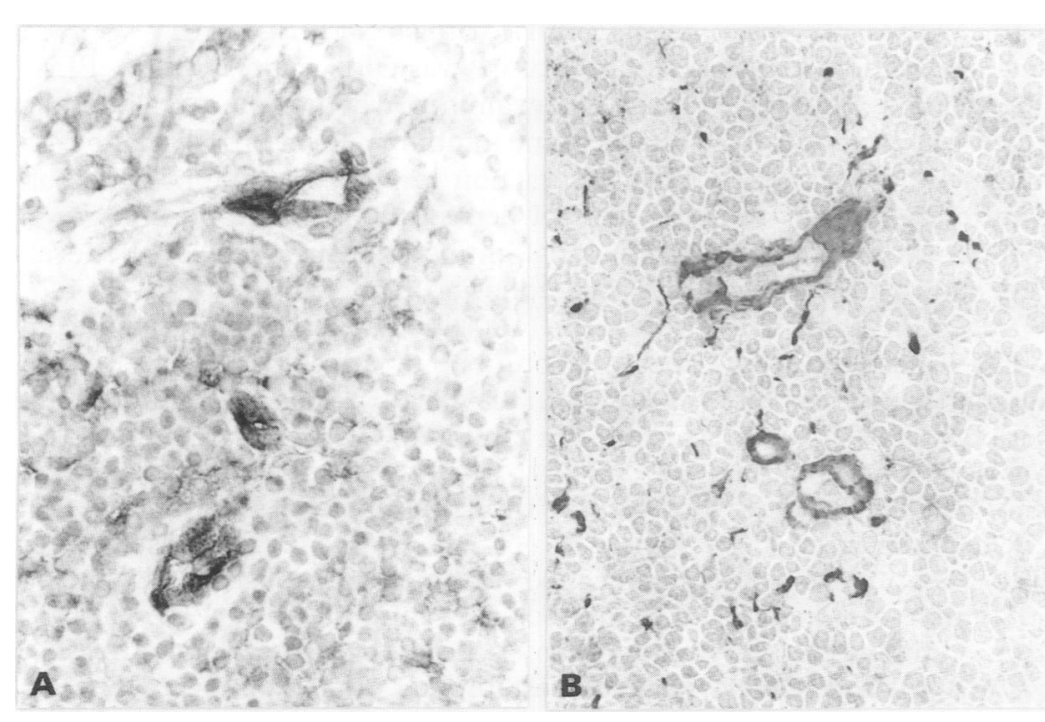

Figure 1 Expression of fibronectin on $(A)$ rheumatoid synovial and $(B)$ peripheral lymph node vessels.

\section{IMMUNOHISTOCHEMISTRY}

Immunoperoxidase staining was performed on $4 \mu \mathrm{m}$ acetone fixed cryostat sections of control non-rheumatoid and rheumatoid synovia and PLNs by an indirect immunoperoxidase procedure as described previously. ${ }^{24}$ The antibody to fibronectin used was a mouse polyclonal antibody. The second step antibody was horseradish peroxidase conjugated goat antimouse immunoglobulin (Dakopatts).

\section{STATISTICS}

To test the difference in in vitro adhesion of lymphocytes in the absence or presence of monoclonal antibodies, Student's $t$ test was used (two-tailed). Values of $\mathrm{p}<0.05$ were considered to be significant.

\section{Results}

EXPRESSION OF FIBRONECTIN ON ENDOTHELIUM IN SYNOVIAL MEMBRANES AND PLNs

Synovectomy specimens from patients with definite or classical RA showing marked lymphocytic infiltrations were selected for study. They contained a spectrum of vessel types; in addition to venules with a flat endothelium, venules lined by endothelial cells, morphologically resembling HEVs in secondary lymphoid organs, were present. All these vessels reacted with the polyclonal antibody against fibronectin (fig 1A), as did the endothelium of the vessels in PLNs (fig 1B). Although the distribution of fibronectin appeared throughout the vessel wall, staining was often more intense at the basement membrane and at the luminal surface of the endothelial cell. The intensity of staining of fibronectin on endothelia in non-rheumatoid synovium was similar to that in PLNs and rheumatoid synovia.

FIBRONECTIN AND CS1 PEPTIDE INHIBIT T LYMPHOCYTE ADHESION TO SYNOVIAL HEV Only marginal $\mathrm{T}$ lymphocyte binding was observed to non-rheumatoid synovial vessels as well as to flat endothelium present in the rheumatoid synovial membrane. The binding of lymphocytes to HEV-like vessels in the synovial membrane of patients with RA ranged from $10 \cdot 2$ to $16 \cdot 8$ per millimetre of HEV. The control peptide CS2 had no effect on lymphocyte adhesion to HEVs compared with lymphocyte binding in the absence of peptide or in the presence of the non-blocking monoclonal antibody CD45. The CS1 peptide significantly abrogated $\mathrm{T}$ cell binding to synovial HEVs (inhibition 54 (5)\%; p<0.005), however. Binding of $\mathrm{T}$ lymphocytes to synovial HEVs was also strongly inhibited by polyclonal antibody to fibronectin (inhibition 51 (4) \% $\mathrm{p}<0.005$ ) (fig 2). The monoclonal antibody to VCAM-1 inhibited $\mathrm{T}$ cell binding to synovial HEVs by $46(2) \%(p<0.005)$. The inhibition of lymphocyte adhesion by VCAM-1 (46 $(2) \%$ ) was further augmented by the 


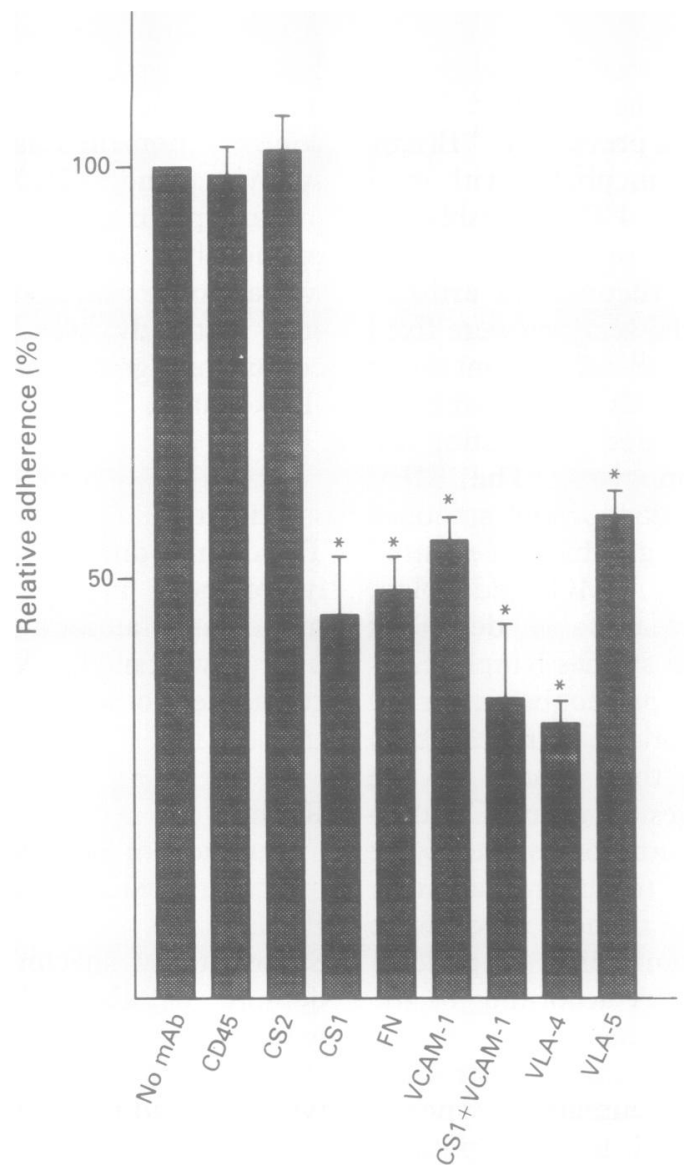

Figure 2 Inhibition of adhesion of normal peripheral blood $T$ lymphocytes to synovial high endothelial venules ( $H E V s)$ by the synthetic peptide CS1 (peptide derived from the type III connecting segment cell binding domain of fibronectin), antibody to fibronectin (FN) and by monoclonal antibodies ( $m A$ bs) against vascular cell adhesion molecule-1

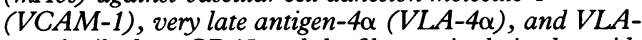
$5 \alpha$. Antibody to CD45 and the fibronectin derived peptide CS2 were used as controls. The adhesion of peripheral blood $T$ cells to synovial HEVs was measured in the presence of either $m A b$ s or single peptides or combinations of $m A b s$ and peptides. Adhesion in the absence of $m A b s$ or peptides (control) is expressed as $100 \%$. Results are expressed as mean ( $S E)$ adhesion ratios $(n \geqslant 3)$. Stars $(*)=$ significantly different from controls $(p<0.05)$. Differences between individual groups are given in the text. The observed lymphocyte adhesion in control slides ranged from $10 \cdot 2$ to 16.8 lymphocytes $/ \mathrm{mm} \mathrm{HEV}$.

addition of CS1 (65 (7)\% compared with inhibition by antibody to VCAM-1 only; $\mathrm{p}=0.05)$. Similar strong inhibition was obtained by blocking the shared counterstructure VLA- 4 by monoclonal antibody to VLA-4 $\alpha$ (68 (2)\%). As might be expected, a combination of CS1 with antibody to VLA- $4 \alpha$ did not further reduce lymphocyte adhesion (inhibition 64 (1)\%; data not shown). Blocking the classical fibronectin receptor with the monoclonal antibody to VLA- $5 \alpha$ also reduced lymphocyte binding, although to a lesser extent than the monoclonal antibody to VLA-4 $\alpha$ (inhibition $42(3) \% ; \mathrm{p}<0 \cdot 01)$. A combination of monoclonal antibodies to VLA-4 and VLA5 showed no additive effects (inhibition 60 (5)\%; data not shown).

Thus the maximum inhibition of binding could be observed with a combination of monoclonal antibody to VCAM-1 with CS1 or when the monoclonal antibody to VLA- $4 \alpha$ was used alone.

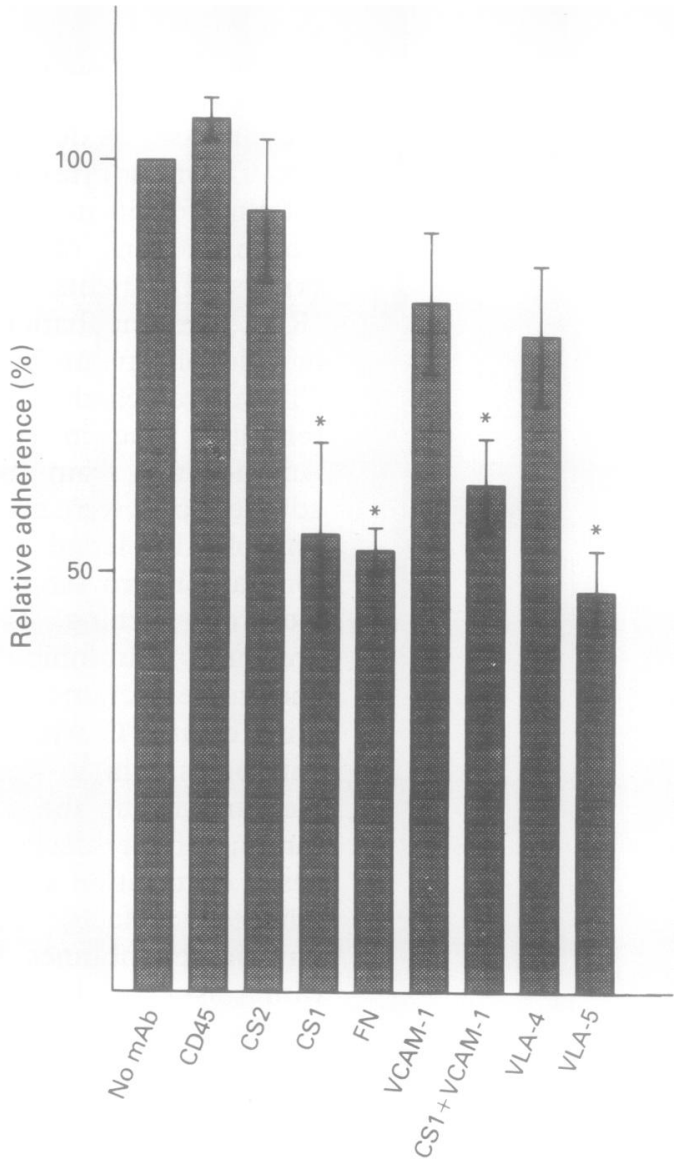

Figure 3 Inhibition of adhesion of normal peripheral blood Tlymphocytes to peripheral lymph node high endothelial venules (HEVs) by the synthetic peptide CS1 (peptide derived from the type III connecting segment cell binding domain of fibronectin), antibody to fibronectin (FN) and by monoclonal antibodies ( $m A b s$ ) against vascular cell adhesion molecule-1 (VCAM-1), very late antigen $-4 \alpha$

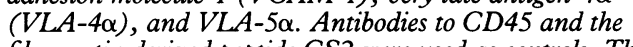
fibronectin derived peptide CS2 were used as controls. The adhesion of peripheral blood $T$ cells to peripheral lymph node $H E V$ was measured in the presence of either $m A b$ or single peptides or combinations of $m A$ bs and peptides. Adhesion in the absence of $m A$ bs or peptides (control) is expressed as $100 \%$. Results are mean (SE) adhesion ratios $(n \geqslant 3)$. Stars $\left(^{*}\right)=$ significantly different from controls $(p<0.05)$.

FIBRONECTIN AND CS1 PEPTIDE INHIBIT T LYMPHOCYTE ADHESION TO PLN HEV

As fibronectin was also expressed on PLN HEVs we examined whether the CS1 peptide had the same inhibitory effect on $\mathrm{T}$ lymphocyte HEV interaction in PLNs. In the presence of the control peptide CS2 or the non-blocking monoclonal antibody against CD45, the level of $\mathrm{T}$ cell adhesion ranged from 13.9 to 14.7 / mm HEV $(100 \%)$. The CS1 peptide reduced the total number of adherent $T$ cells by 45 (9)\% ( $\%<0.005$ ) (fig 3). Also, the antibody to fibronectin inhibited $\mathrm{T}$ lymphocyte binding by $47 \quad(2) \% \quad(p<0.005)$. In contrast, neither blocking with monoclonal antibodies to VCAM-1 or VLA- $4 \alpha$ reached statistical significance, as noted previously. Addition of the monoclonal antibodies to VCAM-1 or VLA-4 $\alpha$ to CS 1 did not further augment CS1induced inhibition of lymphocyte binding (inhibition 39 (6) and 37 (2)\% respectively; fig 3 and data not shown). On the other hand, 
monoclonal antibody to VLA-5 $\alpha$ reduced the lymphocyte adhesion to 48 (5)\% $(\mathrm{p}<0.01)$. Thus the maximum level of inhibition was obtained by CS1 and the antibodies to fibronectin and VLA-5 $\alpha$.

\section{Discussion}

Previously we have shown that the VLA4/VCAM-1 pathway mediates peripheral blood lymphocyte adhesion to inflamed rheumatoid synovium whereas L-selectin (LECAM-1) and CD44 are of major importance in the adhesion of peripheral blood lymphocytes to PLN HEVs. ${ }^{14}$ Using a novel in vitro model of binding of lymphocytes to HEVs in rats, Ager and Humphries showed that lymphocyte adhesion to the surface of cultured PLN-HEV cells can also be inhibited by the CS1 motif in the IIICS of fibronectin. ${ }^{19}$ On this basis they suggested that the ligand on HEVs that could be inhibited by CS1 might be a cell surface form of fibronectin. The aim of this study was to determine whether the CS1 peptide also affects the binding of peripheral blood lymphocytes to HEVs in PLNs and endothelium in inflamed rheumatoid synovium in humans.

Fibronectin staining of endothelium was often most intense at the basal lamina and at the endothelial surface in inflamed synovium and PLNs as described previously. ${ }^{25}$ The present finding that fibronectin supports the adhesion of peripheral blood $T$ cells to synovial and PLN-HEVs supports the view that fibronectin is localised at the upper surface of HEVs. The additional observation that the CS1 motif in IIICS of fibronectin also blocked lymphocyte adhesion to HEVs shows that CS1 is part of the locally expressed fibronectin. Fibronectin is a heterodimer with two cell binding domains - that is, the central cell binding domain containing the RGD sequence important in VLA-5 dependent binding, ${ }^{26}$ and the IIICS domain containing the CS1 motif mediating VLA-4 dependent adhesion of lymphocytes. ${ }^{16} 17$ VLA- $4 \alpha$, a member of the $\beta_{1}$-integrin family of adhesion molecules, has also been implicated as a receptor for VCAM1 , a vascular cell adhesion molecule induced on endothelial cells by tumour necrosis factor $\alpha$, interleukin 1 and lipopolysaccharides. ${ }^{8} 91523$ On the basis of this it was concluded that the VLA-4/VCAM-1 pathway might also play a part in lymphocyte recruitment to inflammatory sites. Indeed, direct evidence for the role of the VLA-4/VCAM-1 pathway in lymphocyte synovial-HEV interaction was reported $^{14}$ and confirmed here. The results obtained here extend these findings to show that VLA-4 mediates lymphocyte binding to synovial HEV not only through interaction with VCAM-1, but also with the CS1 motif of fibronectin. Inhibition of $\mathrm{T}$ cell binding to synovial HEVs by the monoclonal antibody to VLA-4 was significantly stronger $(p<0.05)$ than by the monoclonal antibody to VCAM-1. This difference in inhibition might be explained by the fact that the epitope recognised by the monoclonal antibody to
VLA-4 HP2/1 affects binding to VCAM-1 and the CS1 domain of fibronectin. ${ }^{9}$ This is further supported by our finding that CS1 has no enhancing effect on the monoclonal antibody to VLA-4 HP2/1-induced adhesion blocking. Monoclonal antibodies directed against VLA-5 $\alpha$, which have previously been shown to inhibit cell adhesion to fibronectin, ${ }^{26}$ also showed inhibition of lymphocyte synovial HEV adhesion. This finding, together with the blocking effect of antibodies to fibronectin on lymphocyte synovial endothelium binding, confirms that fibronectin affects lymphocyte immigration in rheumatoid synovium. The fact that combination of VLA-4 and VLA-5 monoclonal antibodies did not completely inhibit lymphocyte adhesion to synovial HEVs suggests that still additional adhesion mechanisms are operational in this binding, or that the molecules operate in a sequence. The CS1 peptide and monoclonal antibody to VLA-5 $\alpha$ also interfered with $\mathrm{T}$ cell binding to PLN-HEV. This finding is consistent with results of Ager and Humphries ${ }^{19}$ and Szekanecz $e t a l^{27}$ in a rat model. In the first study, CS1 peptide inhibited lymphocyte adhesion to the surface of cultured rat HEV cells, but had no effect on lymphocyte migration. In the latter study it was found that VLA-5 not only played a part in lymphocyte adhesion to cultured PLN-HEV, but also in lymphocyte transmigration. In contrast with the monclonal antibody to VLA-5 $\alpha$, the monoclonal antibody to VLA-4 only slightly, but not significantly, inhibited $\mathrm{T}$ cell binding to PLN-HEV (fig 3). ${ }^{14}$ This finding suggests that, apart from VLA-4, another as yet unidentified ligand on peripheral blood $\mathrm{T}$ cells may bind to the CS1 peptide of fibronectin. The failure of the monoclonal antibody to VCAM-1 significantly to influence lymphocyte PLN-HEV interaction might be explained by the fact that VCAM-1 was only sporadically detectable on PLN-HEV. Thus these results show that the VLA-4/VCAM-1 pathway is, if at all, of minor importance in this interaction.

In conclusion, the VLA integrin receptor/ ligand interactions (VLA-4/fibronectin, VLA-4/VCAM-1 and VLA-5/fibronectin) identified in our $T$ cell-endothelial cell adhesion assay are consistent with those identified in adhesion studies of $T$ lymphocytes or cell lines to fibronectin ${ }^{16-18} 262829$ and VCAM-1. ${ }^{8} 9152330$ Importantly, fibronectin has been shown to provide a costimulatory signal in T cell proliferation. ${ }^{31}{ }^{32}$ These results indicate that fibronectin may play important pathophysiological parts in lymphocyte-HEV interactions in rheumatoid synovium and peripheral lymphoid tissues via its CS1 domain and its interaction with the VLA-5 integrin receptor.

This work was supported by grant $88 / C R / 032 / 89$ from the Dutch Rheumatism Foundation. We thank Dr E Bloemena for critical reading of the manuscript, Dr A Ager for providing us with synthetic peptides, Drs R van Lier for monoclonal antibody CLB-CD45 and F Sanchez-Madrid for monoclonal antibody HP2/1, J M Harlan and T M Carlos for monoclonal antibody 4B9, Drs $R M$ van Soesbergen and P Oltshorn for the supply of rheumatoid synovial membranes, $\mathrm{Mr} \mathrm{J}$ de Groot for technical assistance, and Mrs C van Rijn for typing the manuscript. 
1 Gowans J L, Knight E L. The route of recirculation of lymphocytes in the Proc $R$ Soc London (Biol) 1964; 159: 257-82.

2 Pals S T, Horst E , Scheper R J, Meijer C J L M. Mechanisms of human lymphocyte migration and their role in the pathogenesis of disease. Immunol Rev 1989; 108: $111-3$.

3 Stoolman L M. Adhesion molecules controlling lymphocyte migration. Cell 1989; 56: 907-10.

4 Gallatin W M, Weissman I L, Butcher E C. A cell-surface molecule involved in the organ-specific homing of lymphocytes. Nature 1983; 304: 30-4.

5 Jalkanen S, Bargatze R F, De Los Toyos J, Butcher E C. Lymphocyte recognition of high endothelium: antibodies to distinct epitopes of an $85-95 \mathrm{kD}$ glycoprotein antigen differentially inhibit lymphocyte binding to lymph node, mucosal, or synovial endothelial cells. F Cell Biol 1987; 105: 983-90.

6 Haskard D O, Cavender D, Beatty P, Springer T A, Ziff M. T-lymphocyte adhesion to endothelial cells: mechanisms demonstrated by anti-LFA-1 monoclonal antibodies. $f$ demonstrated by anti-LFA-1

7 Dustin $M$, Springer T A. Lymphocyte function associated antigen-1 (LFA-1) interaction with intercellular adhesion molecule-1 (ICAM-1) is one of at least three mechanisms for lymphocyte adhesion to cultured endothelial cells. $f$ Cell Biol 1988; 107: 321-31.

8 Osborn L, Hession C, Tizard R, et al. Direct cloning of vascular cell adhesion molecule-1, a cytokine-induced endothelial protein that binds to lymphocytes. Cell 1989 ; 59: 1203-11.

9 Elices M J, Osborn L, Takada Y, et al. VCAM-1 on activated endothelium interacts with the leucocyte integrin VLA-4 at a site distinct from the VLA-4/fibronectin binding site. Cell 1990; 60: 577-84

10 Larson R S, Springer T A. Structure and function of leukocyte integrins. Immunol Rev 1990; 114: 181-6.

11 Springer T A. Adhesion receptors of the immune system. Nature 1990; 346: 425-34.

12 Hamann A, Jablonsky-Westrich D, Duijvesteijn A, et al. Evidence for an accessory role of LFA-1 in lymphocytehigh endothelium interaction during homing. F Immunol 1988; 140: 693-9.

13 Pals S T, den Otter A, Miedema F, et al. Evidence that leucocyte function-associated antigen-1 is involved in recirculation and homing of human lymphocytes via high endothelial venules. F Immunol 1988; 140: 1851-3.

14 Dinther-Janssen van A C H M, Horst E, Koopman G, et al. The VLA-4/VCAM-1 pathway is involved in lymphocyte adhesion to endothelium in rheumatoid symphocyte adhesion to endothelium in

15 Schwartz B A, Wayner E A, Carlos T M, Ochs H D, Harlan $J M$. Identification of surface proteins mediating adherence of CD11/CD18 deficient lymphoblastoid cells to cultured human endothelium. I Clin Invest 1990; 85: 2019-22.

16 Wayner E A, Garcio-Pardo A, Humphries M J, McDonald $J$ A, Carter W G. Identification and characterisation of the T-lymphocyte adhesion receptor for an alternative cell attachment domain (CS1) in plasma fibronectin. $\mathcal{f} \mathrm{Cell}$ Biol 1989; 109: 1321-30.
17 Guan J L, Hynes R O. Lymphoid cells recognize an alternatively spliced segment of fibronectin via the integrin receptor $\alpha 4 \beta 1$. Cell 1990; 60: 53-61.

18 Humphries M J, Akiyama S K, Komoriya A, Olden K, Yamada K M. Identification of an alternatively spliced site in human plasma fibronectin that mediates cell typespecific adhesion. $\mathcal{F}$ Cell Biol 1986; 103: $2637-47$.

19 Ager A, Humphries M J. Use of synthetic peptides to probe lymphocyte-high endothelial cell interactions. Lymphocytes recognize a ligand on the endothelial surface which contains the CS1 adhesion motif. International Immunology 1990; 2: 921-8.

20 Noesel C van, Miedema F, Brouwer M, Rie M A de, Aarden L A van, Lier R A W van. Regulatory properties of LFA$1 \alpha$ and $\beta$ chains in human $T$ lymphocyte activation. Nature 1988; 333: 850-2.

21 Sanchez-Madrid F, Landazuri M O de, Morago G, Cebrian M, Acevedo A, Bernabeu C. VLA-3: a novel polypeptid associated within the VLA molecule complex: cell distribution and biochemical characterization. Eur Immunol 1986; 16: 1343-9.

22 Keizer G D, Te Velde A A, Schwarning R, Figdor C G, Vries $\mathrm{J} E$ de. Role of p150,50 in adhesion, migration, chemotaxis and phagocytosis of human monocytes. Eur f Immunol 1987; 17: 1317-22

23 Carlos T M, Schwartz B R, Kovach N L, et al. Vascular cell adhesion to cytokine-activated cultured human endothelial cells. Blood 1990; 76: 965-72

24 Dinther-Janssen A C H M van, Pals S T, Scheper R J, Breedveld F, Meijer C J L M. Dendritic cells and high endothelial venules in the rheumatoid synovia membrane. F Rheumatol 1990; 17: 11-7.

25 Stecher V J, Kaplan J E, Connolly K, Mielens Z, Saelen $\mathrm{K}$. Fibronectin in acute and chronic inflammation. Arthritis Rheum 1986; 29: 394-9.

26 Pytela R, Pierschbacher D, Ruoslahti E. Identification and isolation of a $140 \mathrm{kD}$ cell surface glycoprotein with properties expected of a fibronectin receptor. Cell 1985 properties

27 Szekanecz Z, Humphries M J, Ager A. Lymphocyte adhesion to high endothelium is mediated by two $\beta$ integrin receptors for fibronectin, $\alpha 4 \beta 1$ and $\alpha 5 \beta 1 . \mathcal{F} \mathrm{Cell}$ Sci 1992; 101: 885-94.

28 Akiyama S K, Nagata K, Yamada K M. Cell surface receptors for extracellular matrix components. Biochin Biophys Acta 1990; 1031: 91-111.

29 Shimizu Y, Seventer G A van, Horgan K J, Shaw S. Regulated expression and function of three $\operatorname{VLA}(\beta 1)$ integrin receptors on T cells. Nature 1990; 345: 250-2.

30 Rice G E, Munro J M, Bevilacqua M P. Inducible cell adhesion molecule-110 (INCAM-110) is an endothelial adhesion molecule-110 (INCAM-110) is an endothelial receptor for lymphocytes. A CD11/CD18-independ

31 Shimizu Y, Seventer G A van, Horgan K J, Shaw S Costimulation of proliferative responses of resting CD4 $T$ cells by the interaction of VLA-4 and VLA-5 with fibronectin or VLA- 6 with laminin. $f$ Immunol 1990; 145: $59-67$

32 Nojima Y, Humphries M J, Mould A P, et al. VLA-4 mediates $C D 3$-dependent $C D 4+T$ cell activation via the CS1 alternatively spliced domain of fibronectin. 7 Exp Med 1990; 172: 1185-92. 